\title{
Factors affecting the preoperative diagnosis of anterior mediastinal cysts.
}

\section{$\operatorname{AUTHOR}(\mathrm{S})$ :}

Gochi, Fumiaki; Omasa, Mitsugu; Yamada, Tetsu; Sato, Masaaki; Menju, Toshi; Aoyama, Akihiro; Sato, Toshihiko; Chen, Fengshi; Sonobe, Makoto; Date, Hiroshi

\section{CITATION:}

Gochi, Fumiaki ... [et al]. Factors affecting the preoperative diagnosis of anterior mediastinal cysts.. General thoracic and cardiovascular surgery 2015, 63(6): 349-353

\section{ISSUE DATE:}

2015-02-11

URL:

http://hdl.handle.net/2433/200689

\section{RIGHT:}

The final publication is available at Springer via http://dx.doi.org/10.1007/s11748-0150530-2.; この論文は出版社版でありません。引用の際には出版社版をご確認ご利用くだ さい。; This is not the published version. Please cite only the published version. 
1 Factors Affecting the preoperative Diagnosis of Anterior Mediastinal Cysts

2

3 Authors

4 Fumiaki Gochi, Mitsugu Omasa, Tetsu Yamada, Masaaki Sato, Toshi Menju, Akihiro

5 Aoyama, Toshihiko Sato, Fengshi Chen, Makoto Sonobe, Hiroshi Date

6

7 Authors' Affiliation

8 Department of Thoracic Surgery, Kyoto University Graduate School of Medicine

954 Shogoin-kawara-cho, Sakyo-ku,

10 Kyoto 606-8507, Japan

11

12 Corresponding author

13 Mitsugu Omasa, MD, $\mathrm{PhD}$

14 Department of Thoracic Surgery, Kyoto University Graduate School of Medicine

1554 Shogoin-kawara-cho, Sakyo-ku, Kyoto 606-8507, Japan

16 Tel: 81-75-751-4975 / Fax: 81-75-751-4974

17 E-mail: omasa@kuhp.kyoto-u.ac.jp

18

19 Key Words: anterior mediastinal cystic disease, diagnostic accuracy, fluid content

20

21 Abbreviations:

$22 \quad \mathrm{CT}=$ computed tomography 
$1 \quad$ MRI = magnetic resonance imaging

2 FDG-PET = fluorodeoxyglucose positron emission tomography

$3 \quad$ ROC $=$ receiver operating characteristic

4 AUC = area under the ROC curve

$5 \quad \mathrm{SD}=$ standard deviation

6

7

8

9

10

11

12

13

14

15

16

17

18

19

20

21

22 


\section{Abstract}

\section{Objective}

3 Although anterior mediastinal cysts are generally benign diseases, the preoperative

4 diagnosis of these lesions is not necessarily accurate. This study aimed to investigate the

$5 \quad$ factors affecting the preoperative diagnosis of anterior mediastinal cysts.

6 Methods

$7 \quad$ We conducted a retrospective analysis of 35 patients with pathologically diagnosed

8 anterior mediastinal cysts (20 thymic cysts, 7 bronchogenic cysts, 5 pericardial cysts,

9 and 3 others) that were resected at our hospital and evaluated their preoperative

10 diagnosis, cyst size, and fluid content.

\section{Results}

12 Eighteen, 15, 1, and 1 patient(s) were preoperatively diagnosed with cystic disease,

13 thymoma, thymic cancer, and teratoma, respectively. Cysts were significantly larger in

14 the correct diagnosis group (40.2 $\pm 18.8 \mathrm{~mm})$ than in the incorrect diagnosis group (21.1

$15 \pm 10.4 \mathrm{~mm})(p=0.0011)$. The cut-off value of the cyst size which separates these

16 groups, as indicated by the receiver operating characteristic curve, was $28 \mathrm{~mm}$, with a

17 sensitivity and specificity of 0.722 and 0.823 , respectively. The diagnostic accuracy for

18 mucinous cysts was significantly lower $(p<0.001)$ than that for serous cysts.

\section{Conclusion}

20 The presence of cysts smaller than $28 \mathrm{~mm}$ and mucinous fluid content were possible

21 factors resulting in inaccurate preoperative diagnosis. 
1

2

3

4

5

6

(1)

\section{Introduction}

The preoperative diagnosis of anterior mediastinal masses depends on radiographic imaging because identification by biopsy can be difficult. However, the diagnostic accuracy of CT and MRI has limited reliability [1]. Although the diagnostic rate for mediastinal cysts has increased in recent years because of advances in imaging modalities, every possible diagnostic imaging modality is not always used for them in the clinical setting because a certain number of mediastinum cysts can be easily diagnosed with CT alone. The aim of this retrospective study was to evaluate the factors affecting the preoperative diagnosis of surgically resected anterior mediastinal cysts.

\section{Subjects}

Between 2001 and 2013, 211 patients underwent surgical resection of anterior mediastinal masses at our hospital. In this study, we included 35 patients (16.6\%) who were histologically diagnosed with primary anterior mediastinal cysts, irrespective of the origin.

\section{Methods}

The following characteristics of each patient were analyzed retrospectively: age, sex, preoperative diagnosis, cyst size, imaging study data (CT, MRI, FDG-PET), and fluid content, as well as the pathological diagnosis. The cyst size was defined as the long span of the fluid-filled cyst just after resection. Patients were divided into the mucinous fluid group and serous fluid group according to the macroscopic findings of cyst fluid content. Patients with turbid or bloody cyst fluid were included in the 
1 mucinous fluid group and those with clear or yellowish transparent fluid were included

2 in the serous fluid group.

3 The preoperative diagnoses were provided by thoracic surgeons and were

4 comprehensively based on clinical features and imaging diagnoses made by the

5 radiologists. T2 high and no enhancement with contrast MRI (if performed) and no or

6 faint FDG uptake were the standard diagnostic criterion for cysts.

Results for continuous variables are presented as the median [ranges] or mean

$8 \pm \mathrm{SD}$ and those for categorical variables are presented as numbers. The Mann-Whitney

9 U test was used to compare the results of continuous variables. The chi-squared test or

10 Fisher's exact test was used to compare the results of categorical variables between the

11 two groups. ROC curve analysis was used to evaluate the diagnostic accuracy and to

identify a cut-off value at a definite level of true sensitivity against false positivity. All

$13 p$-values were two-sided, and $p$-values $<0.05$ were considered statistically significant.

JMP 11 (SAS Institute, Cary, NC) was used for statistical analysis.

University (E1972).

Results

The subjects included 21 men and 14 women aged 20-79 years (median age, 59.0 years). The preoperative diagnoses included 18, 15, 1, and 1 patient(s) with cystic disease, thymoma, thymic cancer, and teratoma, respectively. The average cyst size was $30.9 \mathrm{~mm}$, but the cysts varied widely in size $(5-80 \mathrm{~mm})$. Of these 35 patients, all 
1 patients underwent CT (contrast CT was performed in 12 cases), 17 patients underwent

2 MRI, and 14 patients underwent FDG-PET. The fluid content of the cysts was

3 mucinous, serous, and unknown in 15, 14, and 6 patients, respectively. Concerning the

4 histological etiology, 20, 7, 5, 1, 1, and 1 patient(s) had thymic, bronchogenic,

5 pericardial, parathyroid, lymphoepithelial, and mesothelial cysts, respectively.

6 Additionally, multilocular cysts were identified in 7 patients (6 thymic cysts and 1

7 pericardial cyst), and unilocular cysts were detected in 28 patients. Only 18 patients

8 (51.4\%) were diagnosed correctly with primary mediastinal cystic disease

9 preoperatively, irrespective of the origin (Table 1).

\section{Comparison of the correct and incorrect diagnosis groups}

We defined preoperative diagnoses of primary mediastinal cystic disease irrespective of origin as "correct" and preoperative diagnoses other than primary mediastinal cystic diseases as “incorrect”. The correct group included 18 patients (51.4\%), and the incorrect group included 17 patients (48.6\%). age, presence or absence of enhanced CT, presence or absence of MRI imaging data, FDG accumulation, origin of the cyst, category of the cyst, and oblateness on CT examination (oblateness $=$ diameter crossed at right angles/maximum diameter calculated on the basis of the CT scans). (40.2 $\pm 18.8 \mathrm{~mm}$ in the correct diagnosis group vs. $21.1 \pm 10.4 \mathrm{~mm}$ in the incorrect 
1 diagnosis group, $p=0.001$; (Fig. 1). Serous fluid was observed more frequently in the

2 correct group than the incorrect group $(p<0.001)$. There was also a significant

3 difference between the 2 groups in sex distribution $(p=0.027)$ (Table 2).

4

5

Preoperative diagnostic rate according to cyst size

6

7

We analyzed the diagnostic accuracy according to cyst size using ROC curve analysis. A sensitivity of 0.722 and a false positivity value ( 1 - specificity) of 0.177 comprised the left upper corner of the ROC curve, with the corresponding cut-off value of the cyst size at $28 \mathrm{~mm}$. The area under the ROC curve (AUC) was 0.825 (Fig. 2).

\section{Fluid content in the cyst}

The fluid type was confirmed in 29 of 35 patients, and these patients were retrospectively divided into the mucinous fluid (15 patients) and serous fluid groups (14 patients) according to the type of fluid in the cysts.

The cyst size and oblateness of the cysts (as determined using CT) and the diagnostic accuracy were compared between the 2 groups. Mucinous cysts were significantly smaller $(p=0.026)$ and rounder $(p=0.045)$ than serous cysts. In addition, the accuracy of the preoperative diagnosis for mucinous cysts was significantly lower ( $p$ $<0.001$ ) than that for serous cysts (Table 3).

\section{Diagnosis by cyst size and fluid content with or without contrast CT}


Although there was no significant difference in the diagnostic accuracy

2 regardless of the presence or absence of CT enhancement (Table 2), contrast CT was

3 apparently superior to non-contrast CT in the diagnostic accuracy for cysts under 28

$4 \mathrm{~mm}$ in size or mucinous fluid cysts. Non-contrast CT showed a significantly lower

5 diagnostic rate for cysts under $28 \mathrm{~mm}$ in size or cysts with mucinous fluid content.

6 However, incorrect diagnoses often occurred with cysts under $28 \mathrm{~mm}$ in size, even with

7 contrast CT (Table 4).

8

9 Discussion

Anterior mediastinal cystic disease is an inclusive term describing

11 heterogeneous cystic diseases that originate from various tissues located posterior to the sternum and anterior to the heart and brachiocephalic vessels. Although this disease

13 includes different pathological entities, these entities have overlapping clinical and

14 radiological features.

Anterior mediastinal cystic disease accounts for $20 \%$ of all primary anterior mediastinal diseases and primarily includes thymic, bronchogenic, and pericardial cysts [2]. Differential diagnoses of anterior mediastinal tumors, such as thymic epithelial and germ cell tumors, must be considered. As these anterior mediastinal tumors are often accompanied by cystic lesions [3, 4], the differential diagnosis is often difficult.

Percutaneous needle biopsy is difficult because of the tumor localization. Therefore, the pretreatment diagnosis is often dependent on the radiographic findings. 
2 symptoms, large cysts can cause dyspnea as a result of tumor compression [5].

3 Symptomatic cysts, suspected malignancy, cyst infection, tracheal compression,

4 progressive growth, presence in children, and atypical location or characteristics have

5 been proposed as criteria that signal the requirement of surgical intervention.

6 Notwithstanding, it remains controversial whether asymptomatic unilocular cysts can be

$7 \quad$ observed or resected, as malignancy cannot be ruled out by imaging [6, 7].

For these reasons, the pretreatment diagnosis of anterior mediastinal cysts

9 using imaging methods is essential. The diagnostic accuracy of thymic cysts is reported not to exceed 63\% using the combined application of CT and MRI [1]. The diagnostic accuracy using all diagnostic imaging methods is high. In the clinical setting, however, every imaging method is not always selected because some cystic cases can be diagnosed easily with only plain chest CT. Therefore, it is important to investigate factors affecting preoperative diagnosis.

First, we focused on cyst size and analyzed the preoperative diagnostic accuracy according to cyst size using ROC curves to select the cut-off point. Because the AUC of 0.825 was indicative of moderate accuracy for this standard [8], cyst size was determined to be a significant factor affecting the preoperative diagnostic accuracy.

The cut-off point for cyst size deduced using ROC curve analysis was $28 \mathrm{~mm}$ more often diagnosed inaccurately. 
We then focused on the fluid content of the cysts. Mucinous cysts are smaller,

2 rounder, and more difficult to diagnose than serous cysts. We speculate that the

3 morphological similarity to solid tumors due to the increased viscosity and

4 concentration of fluid is responsible for the low diagnostic accuracy of mucinous cysts.

5 MRI, FDG-PET, and enhanced CT are performed in a limited number of patients in our

6 department. MRI is a valid modality for cyst diagnosis [1]. Although there was no

7 difference in the diagnostic accuracy with the inclusion or exclusion of MRI in this

8 study, we believe that selection bias was the reason for this result. Regarding FDG-PET,

9 its diagnostic value has not been fully clarified. It has been reported that FDG uptake by thymic cysts is low, which is in contrast to the high FDG uptake by high-risk thymomas

$11[9,10]$. On the contrary, FDG uptake can be observed in patients with normal thymus or thymic hyperplasia [11], and no FDG uptake was occasionally observed in patients with low-risk thymoma. As our result suggested, FDG-PET is merely an auxiliary diagnostic modality for anterior mediastinal cysts at this time. Contrast CT was apparently superior to non-contrast CT in the diagnostic accuracy for cysts under $28 \mathrm{~mm}$ in size or cysts with mucinous fluid content. Although contrast CT should be selected especially for these cases, we must remember that incorrect diagnoses often occur for cysts under 28 $\mathrm{mm}$ in size, even with contrast CT. congenital, and the second is multilocular and acquired. Multilocular cysts are 
1 no difference in the diagnostic accuracy for unilocular and multilocular cysts in our

2 study.

3

4

5

6

All the operated cases $(n=17)$ with preoperative cyst diagnoses in our institution were also pathologically cysts. Although we could not evaluate the diagnostic accuracy of preoperative cyst diagnoses because most of cases with the preoperative cyst diagnosis had been observed without surgery, we believe the preoperative cyst diagnosis has a high diagnostic accuracy.

The diagnostic accuracy depends on the performance of medical device.

However, in our study, the diagnostic accuracy (0.58) in the former period (2001-2007) was not inferior to that (0.50) in the latter period (2008-2013).

\section{Study limitation}

In this retrospective study, the selection of diagnostic imaging methods was left to each doctor's judgment. Because we did not evaluate the true sensitivity or specificity, the values obtained are a rough estimate.

\section{Conclusion}

We examined the preoperative diagnosis of surgically resected primary anterior mediastinal cysts. The preoperative diagnostic accuracy for anterior primary mediastinal cysts was relatively low. The presence of cysts smaller than $28 \mathrm{~mm}$ and mucinous fluid content were possible factors resulting in inaccurate preoperative diagnosis.

\section{Conflict of interest}


1 The authors of this manuscript have no conflicts of interest to disclose.

2

3

4

5

6

7

8

9 


\section{References}

3

4 1. Tomiyama N, Honda O, Tsubamoto M, Inoue A, Sumikawa H, Kuriyama K, et al. Anterior

5 mediastinal tumors: diagnostic accuracy of CT and MRI. Eur J Radiol. 2009;69(2):280-8.

62 Strollo DC, Rosado-de-Christenson ML, Jett JR. Primary mediastinal tumors: part II. Tumors

$7 \quad$ of the middle and posterior mediastinum. Chest. 1997;112(5):1344-57.

$83 . \quad$ Poorabdollah M, Mehdizadeh E, Mohammadi F, Sabeti S. Metaplastic thymoma: report of an

9 unusual thymic epithelial neoplasm arising in the wall of a thymic cyst. International journal of surgical 10 pathology. 2009;17(1):51-4.

114 Moran CA, Suster S. Thymoma with prominent cystic and hemorrhagic changes and areas of necrosis and infarction: a clinicopathologic study of 25 cases. The American journal of surgical pathology. 2001;25(8):1086-90.

$14 \quad$ 5. Zambudio AR, Lanzas JT, Calvo MJ, Fernandez PJ, Paricio PP. Non-neoplastic mediastinal

15 cysts. European journal of cardio-thoracic surgery : official journal of the European Association for

16 Cardio-thoracic Surgery. 2002;22(5):712-6.

17 6. Kirmani B, Sogliani F. Should asymptomatic bronchogenic cysts in adults be treated

18 conservatively or with surgery? Interact Cardiovasc Thorac Surg. 2010;11(5):649-59.

$197 . \quad$ Cohen AJ, Thompson L, Edwards FH, Bellamy RF. Primary cysts and tumors of the 20 mediastinum. Ann Thorac Surg. 1991;51(3):378-84; discussion 85-6.

$21 \quad$ 8. Fischer JE, Bachmann LM, Jaeschke R. A readers' guide to the interpretation of diagnostic

22 test properties: clinical example of sepsis. Intensive Care Med. 2003;29(7):1043-51. 
19 Gumar A, Regmi SK, Dutta R, Kumar R, Gupta SD, Das P, et al. Characterization of thymic

2 masses using (18)F-FDG PET-CT. Ann Nucl Med. 2009;23(6):569-77.

3 10. Shibata H, Nomori H, Uno K, Sakaguchi K, Nakashima R, Iyama K, et al.

$4 \quad$ 18F-fluorodeoxyglucose and 11C-acetate positron emission tomography are useful modalities for

5 diagnosing the histologic type of thymoma. Cancer. 2009;115(11):2531-8.

6 11. Benveniste MF, Rosado-de-Christenson ML, Sabloff BS, Moran CA, Swisher SG, Marom

7 EM. Role of imaging in the diagnosis, staging, and treatment of thymoma. Radiographics.31(7):1847-61;

8 discussion 61-3.

9 12. Suster S, Rosai J. Multilocular thymic cyst: an acquired reactive process. Study of 18 cases.

10 The American journal of surgical pathology. 1991;15(4):388-98.

11 13. Nakamura S, Tateyama H, Taniguchi T, Ishikawa Y, Kawaguchi K, Fukui T, et al.

12 Multilocular thymic cyst associated with thymoma: a clinicopathologic study of 20 cases with an

13 emphasis on the pathogenesis of cyst formation. The American journal of surgical pathology.

14 2012;36(12):1857-64. 
Table 1. Patient characteristics

\begin{tabular}{|c|c|}
\hline Variables & Number [range] \\
\hline Sex $(M / F)$ & $21 / 14$ \\
\hline Age, y [range] & 59 [20-79] \\
\hline \multicolumn{2}{|l|}{ Preoperative diagnosis } \\
\hline Cystic disease & 18 \\
\hline Thymoma & 15 \\
\hline Thymic carcinoma & 1 \\
\hline Teratoma & 1 \\
\hline Cyst size, mm [range] & $30.9[5-80]$ \\
\hline CT (performed/not performed) & $35 / 0$ \\
\hline with contrast/without contrast & $12 / 23$ \\
\hline MRI (performed/not performed) & $17 / 18$ \\
\hline FDG-PET (performed/not performed) & $14 / 21$ \\
\hline \multicolumn{2}{|l|}{ Fluid content } \\
\hline Mucinous & 15 \\
\hline Serous & 14 \\
\hline Unknown & 6 \\
\hline \multicolumn{2}{|l|}{ Accuracy of preoperative diagnosis } \\
\hline Correct/ Incorrect & $18 / 17$ \\
\hline \multicolumn{2}{|l|}{ Pathology } \\
\hline Thymic cyst & 20 \\
\hline Bronchogenic cyst & 7 \\
\hline Pericardial cyst & 5 \\
\hline Lymphoepithelial cyst & 1 \\
\hline Parathyroid cyst & 1 \\
\hline Mesothelial cyst & 1 \\
\hline \multicolumn{2}{|l|}{ Category of cyst } \\
\hline Unilocular cyst & 28 \\
\hline Multilocular cyst & 7 \\
\hline
\end{tabular}


Table 2. Relation of variables with correct and incorrect preoperative diagnoses

\begin{tabular}{|c|c|c|c|}
\hline & Correct $(n=18)$ & Incorrect $(n=17)$ & $p$-value \\
\hline Sex & & & 0.027 \\
\hline Men & 14 & 7 & \\
\hline Women & 4 & 10 & \\
\hline Age (y) & $55.7 \pm 17.0$ & $55.7 \pm 15.2$ & 0.716 \\
\hline Cyst size (mm) & $40.2 \pm 18.8$ & $21.1 \pm 10.4$ & 0.001 \\
\hline Fluid content & & & $<0.001$ \\
\hline Mucinous fluid & 3 & 12 & \\
\hline Serous fluid & 13 & 1 & \\
\hline CT enhancement & & & 0.725 \\
\hline with contrast & 7 & 5 & \\
\hline without contrast & 11 & 12 & \\
\hline MRI & & & 0.862 \\
\hline Performed & 9 & 8 & \\
\hline Not performed & 9 & 9 & \\
\hline FDG-PET accumulation & & & 0.801 \\
\hline Negative & 6 & 5 & \\
\hline Positive & 2 & 1 & \\
\hline Not performed & 11 & 10 & \\
\hline Pathology & & & 0.010 \\
\hline Thymic cyst & 9 & 11 & \\
\hline Bronchogenic cyst & 2 & 5 & \\
\hline Pericardial cyst & 4 & 1 & \\
\hline Miscellaneous & 3 & 0 & \\
\hline Category of cyst & & & 0.735 \\
\hline Unilocular cyst & 14 & 14 & \\
\hline Multilocular cyst & 4 & 3 & \\
\hline Oblateness on CT & 0.675 & 0.658 & 0.770 \\
\hline
\end{tabular}

Oblateness on CT = Diameter crossed at right angle / maximum diameter on CT 
Table 3. Characteristics of the fluid content of the cyst

\begin{tabular}{lccc}
\hline & Mucinous $(\mathbf{n}=\mathbf{1 5})$ & Serous $(\mathbf{n}=\mathbf{1 4})$ & $\boldsymbol{p}$-value \\
\hline Size $(\mathrm{mm})$ & $24.7 \pm 13.5$ & $40.9 \pm 20.4$ & 0.026 \\
Oblateness on CT & $0.739 \pm 0.164$ & $0.614 \pm 0.138$ & 0.045 \\
Diagnosis & & & $<0.001$ \\
Correct & 3 & 13 & \\
Incorrect & 12 & 1 & \\
\hline
\end{tabular}

Oblateness on CT = Diameter crossed at right angle / maximum diameter on CT 
Table 4. Diagnosis by cyst size and fluid content with or without contrast CT

\begin{tabular}{|c|c|c|c|}
\hline & With contrast & Without contrast & $p$-value* \\
\hline \multicolumn{4}{|l|}{ Cyst size } \\
\hline \multicolumn{4}{|l|}{ Cyst size $\geq 28 \mathrm{~mm}$} \\
\hline correct/ incorrect & $4 / 0$ & $9 / 3$ & \multirow{3}{*}{0.012} \\
\hline Cyst size $\prec 28 \mathrm{~mm}$ & & & \\
\hline correct/ incorrect & $3 / 5$ & $2 / 9$ & \\
\hline \multicolumn{4}{|l|}{ Fluid content } \\
\hline \multicolumn{4}{|l|}{ Serous fluid } \\
\hline correct/ incorrect & $4 / 1$ & $9 / 0$ & \multirow{3}{*}{$<0.001$} \\
\hline Mucinous fluid & & & \\
\hline correct/ incorrect & $2 / 1$ & $1 / 11$ & \\
\hline
\end{tabular}




\section{Figure Legends}

\section{Figure 1}

Preoperative diagnosis according to cyst size. Cysts were significantly ( $p=0.0011$ ) larger in the correct diagnosis group than in the incorrect diagnosis group.

Figure 2

A sensitivity of 0.722 and a false positivity value ( 1 - specificity) of 0.177 comprised the left upper corner of the curve (marked) with the corresponding cut-off value of the cyst size $(28 \mathrm{~mm})$. The area under the receiver operating characteristic curve was 0.825 . 
Fig. 1 Preoperative diagnosis according to cyst size

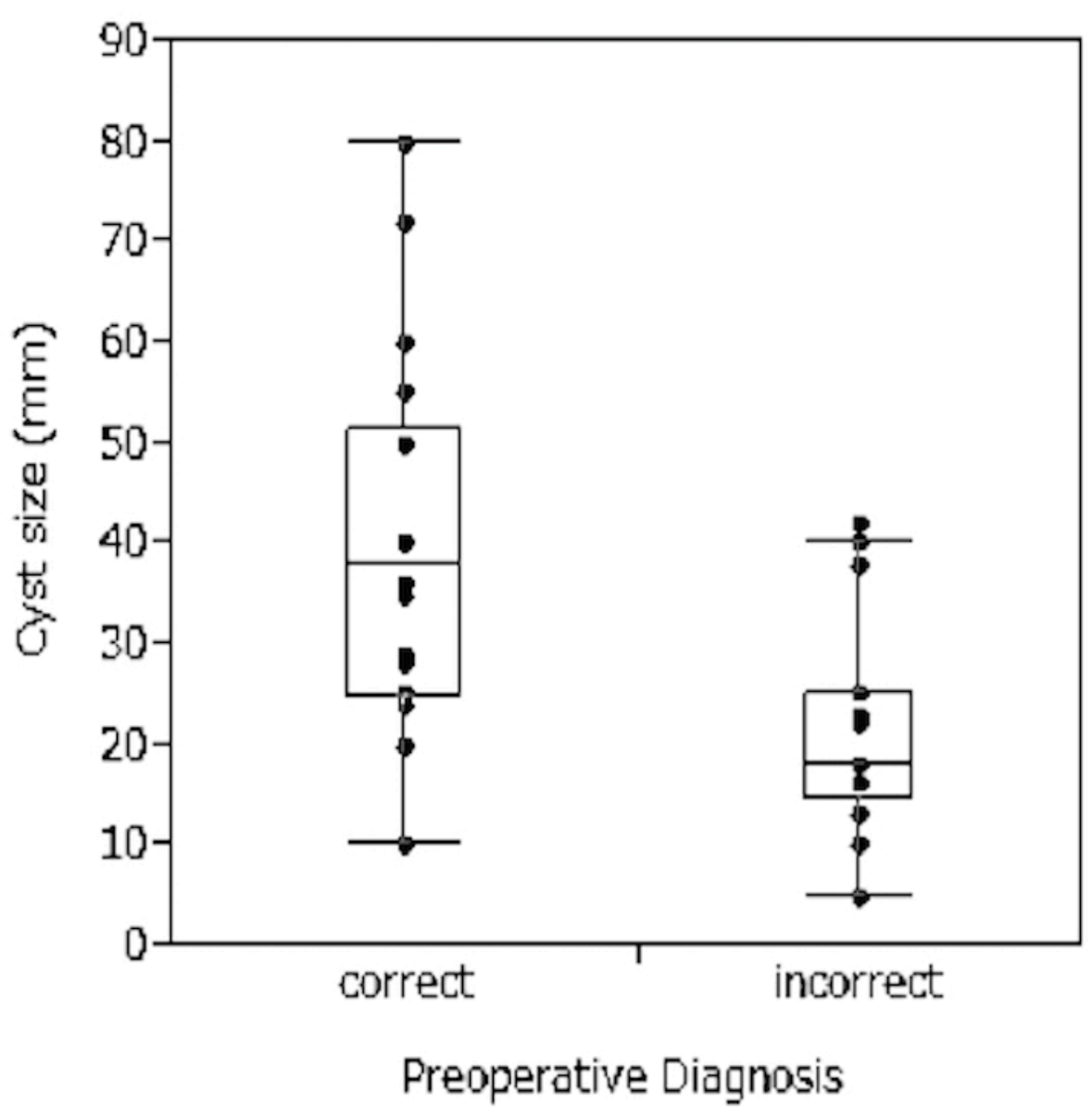


Fig. 2 Receiver operating characteristic curve analysis

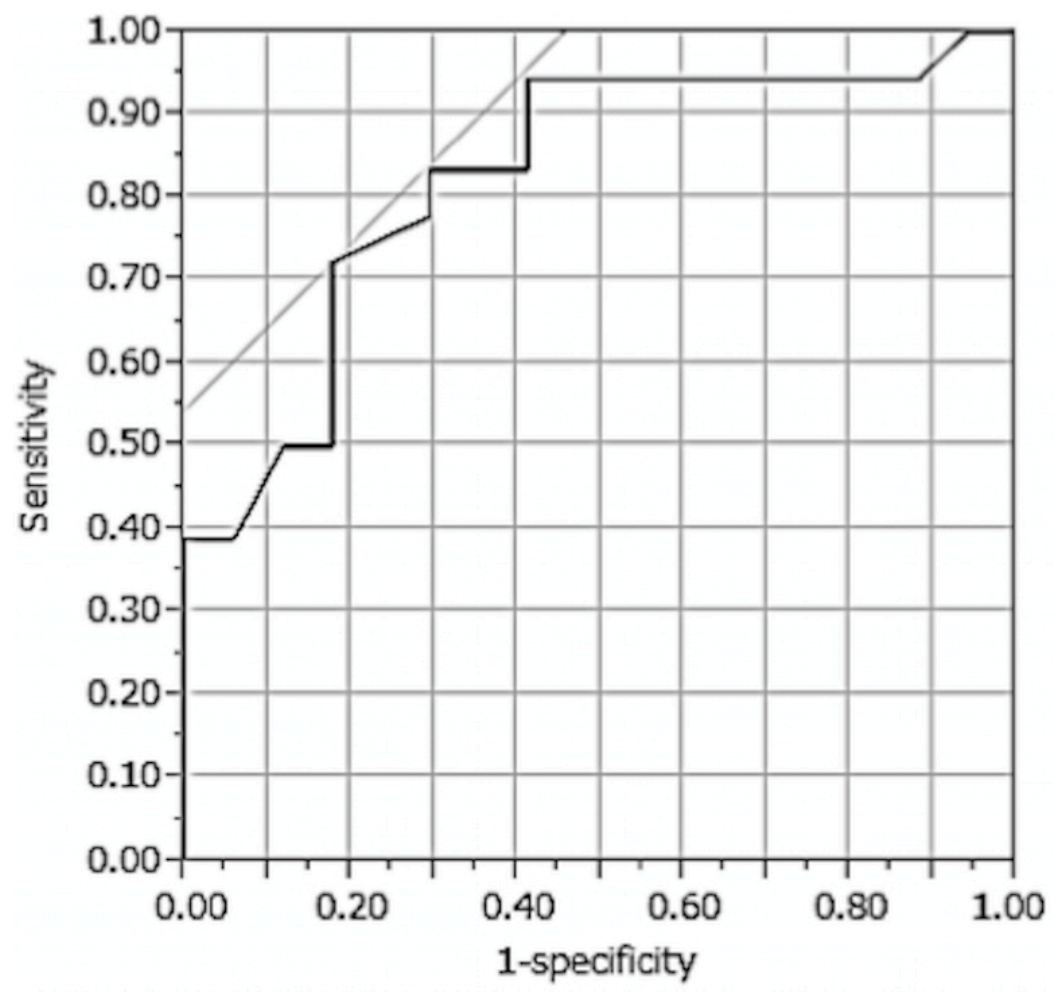

